\title{
Ultrasound imaging and beyond: recent advances in medical ultrasound
}

\author{
Jongbum Seo ${ }^{1}$ Young-sun $\mathrm{Kim}^{2}$
}

Received: 4 April 2017/Revised: 9 April 2017/Accepted: 10 April 2017/Published online: 14 April 2017

(C) Korean Society of Medical and Biological Engineering and Springer 2017

Medical ultrasound represented by ultrasound imaging is generally believed to be well-known area in research, because ultrasound imaging systems has been widely distributed and actively used in the world since the 1960s. The annual world-wide use of ultrasound imaging in practice is already over billions and all pregnant women have been screened with ultrasound in some countries, such as Germany, Norway, Iceland, and Austria for years [1]. In industry, not only portable systems were available since the early 2000s, but also wireless ultrasound imaging transducers cooperating with tablet PCs were introduced in the early 2010s. Accordingly, ultrasound in medicine has been believed relatively low-tech and obsolete research field to general audience.

However, medical ultrasound has been one of the most active research areas for last 20 years and numerous outstanding break-troughs has been reported in both diagnostic and therapeutic ultrasound. In ultrasound imaging, elastography, ultrasound contrast agent imaging [2], super resolution imaging, and 2D array transducer are noteworthy. Elastic property of a given tissue can be critical for cancer diagnosis of a certain fibrous tumor and evaluation of vulnerable plaque in artery during stent implantation [3]. However, elastic characteristics cannot be clearly

Jongbum Seo

jongbums@yonsei.ac.kr

$\triangle$ Young-sun Kim

jeants.kim@gmail.com

1 Department of Biomedical Engineering, Yonsei University, 304 Medical Industry Techno Tower, Wonju, Gangwon 220-710, Republic of Korea

2 Uterine Fibroid Integrated Management Center, MINT Intervention Hospital, Seoul 26493, Republic of Korea visualized in general ultrasound images. In order to quantitative measure of elasticity, radiation force which provides controlled deformation force on target tissue was implemented by ultrasound imaging transducer and speckle tracking algorithm which provides means of local tissue position tracking up to tens of micrometers was developed. These technologies later combined with a high frame late ultrasound imaging system whose frame late can reach up to $10,000 / \mathrm{s}$ and presented high resolution quantitative elastography [4, 5]. On the other hand, super resolution imaging was achieved by simply tracking microbubbles in blood vessel with a high frame late system introduced above [6]. Since a single microbubble can be counted as a scatterer whose resolution is several orders higher than the point spread function of ultrasound system, the resultant images can show us excellent vasculature networks in deeply located organ. In order to expand these new technologies of ultrasound imaging in 3D, 2D array transducer is required, so that micro-machined transducers were also developed [7].

While researches and developments in ultrasound imaging area have been outstanding recently, novel approaches in therapeutic ultrasound have been equally impressive if not superior. 2D phased array transducers in high intensity focused ultrasound (HIFU) not only facilitate fast abdominal cancer treatment [8] but also enable to treat brain diseases without craniotomy or burr hole trephination. With the help of MRI guidance for brain treatment, ultrasound technology brought non-invasive means for selective treatment brain related disease and the result from clinical trials in tremor patients was exceptionally promising [9, 10]. Spatially targeted drug delivery with ultrasound and microbubbles is another hot issue in medical ultrasound. Ultrasound is time varying acoustic pressure field and microbubbles in ultrasound field oscillate 
and/or rapidly collapse. These bubble activities are generally defined as acoustic cavitation. If cavitation activities occur nearby biological boundary which acts as a barrier, the barrier function of the boundary is temporally disturbed. Combined with the spatially selective nature of focused ultrasound, ultrasound mediated spatially targeted drug delivery with microbubbles has been a main research topic over last 20 years. As for the application, ultrasound mediated drug delivery through blood brain barrier (BBB) has been most successful in animal model experiments $[11,12]$. Although BBB opening is caught attention from general public, ultrasound mediated drug delivery has also been widely researched for the targeted cancer treatment in both body and brain. The last noteworthy ultrasound therapy is histotripsy [13]. This term is antonym to lithotripsy which indicates destruction of calcified targets such as kidney stone. In histotripsy, the target soft tissue is completely pulverized with multiple cavitations induced by repetitive high amplitude ultrasound pulses without cavitation seeds. This technique provides clear cut lines between treated tissue and surrounding tissue unlike HIFU which generally uses thermal effect and has smeared boundary. Accordingly, histotripsy can be an extremely selective tool for cancer treatment.

Although it is not mentioned above, there are additional ultrasound related research areas such as photoacoustics and ultrasound neuromodulation. Photoacoustic imaging (PAI) uses a pulsed laser excitation to generate sound in body and receives the signal with an ultrasound transducer. Since the focal spot size of laser is multiple orders smaller than that of ultrasound beam, the image resolution is phenomenally higher compared to general ultrasound images. PAI can be also easily implemented with ultrasound imaging since they can share the identical transducer [14]. Additionally, most of algorithms developed for ultrasound imaging over the decades can be directed translated to PAI and ultrasound elastography could be one of the top candidates. While most of the introduced topics have at least a specific application, ultrasound neuromodulation is in a still early stage of research to determine a certain definitive application. Since the middle of 2000s, both excitational and inhibitive neural activities induced by low intensity ultrasound have been demonstrated in the number of animal models [15]. Although the underlying mechanism has not yet been clearly understood, many of researchers expect that ultrasound neuromodulation might replace transcranial magnetic stimulation (TMS).

As described above, medical ultrasound field is boarder than general perception at least in research area. Hence, this special issue is designed to bring in various ultrasoundrelated topics in medicine. We also tried to invite both engineering and medical sectors to include different perspectives in medical ultrasound. Although we could not cover all the topics mentioned above, articles in this special issue will extend the general perception and will provide broader understanding on ultrasound research field.

Finally, we guest editors would like to express our gratitude to the authors who contributed in this special issue in the field of medical ultrasound.

\section{References}

1. Szabo TL. Diagnostic ultrasound imaging inside out. Amsterdam: Elsevier Academic Press; 2004.

2. Lee H, Kimm H, Han H, Lee M, Lee S, Yoo H, Chang JH, Kim $\mathrm{H}$. Microbubbles used for contrast enhanced ultrasound and theragnosis: a review of principles to applications. Biomed Eng Lett. 2017. doi:10.1007/s13534-017-0016-5.

3. Kwon SJ, Jeong MK. Advances in ultrasound elasticity imaging. Biomed Eng Lett. 2017. doi:10.1007/s13534-017-0014-7

4. Sandrin L, Tanter M, Catheline S, Fink M. Shear modulus imaging with $2 \mathrm{~d}$ transient elastography. IEEE Trans UFFC. 2002;49:426-35

5. Bruce M, Kolokythas O, Ferraioli G, Filice C, O'Donnell M. Limitations and artifacts in shear-wave elastography of the liver. Biomed Eng Lett. 2017. doi:10.1007/s13534-017-0028-1

6. Errico C, Pierre J, Pezet S, Desailly Y, Lenkei Z, Couture O, Tanter M. Ultrafast ultrasound localization microscopy for deep super-resolution vascular imaging. Nature. 2015;527:499-502.

7. Lee W, Roh Y. Ultrasonic transducers for medical diagnostic imaging. Biomed Eng Lett. 2017. doi:10.1007/s13534-017-0021-8.

8. Kim Y. Clinical application of high-intensity focused ultrasound ablation for uterine fibroids. Biomed Eng Lett. 2017. doi:10.1007/ s13534-017-0012-9.

9. Elias WJ, Lipsman N, Ondo WG, Ghanouni P, Kim YG, Lee W, Schwartz M, Hynynen K, Lozano AM, Shah BB, Huss D, Dallapiazza RF, Gwinn R, Witt J, Ro S, Eisenberg HM, Fishman PS, Gandhi D, Halpern CH, Chuang R, Pauly KB, Tierney TS, Hayes MT, Cosgrove GR, Yamaguchi T, Abe K, Taira T, Chang JW. A randomized trial of focused ultrasound thalamotomy for essential tremor. N Engl J Med. 2016;375:730-9.

10. Chang WS, Chang JW. Focused ultrasound treatment for central nervous system diseases: neurosurgeon's perspectives. Biomed Eng Lett. 2017. doi:10.1007/s13534-017-0013-8.

11. Choi JJ, Pernot M, Small SA, Konofagou EE. Noninvasive, transcranial and localized opening of the blood-brain barrier using focused ultrasound in mice. Ultrasouind in Med Biol. 2007;33:95-104.

12. Han M, Hur Y, Hwang J, Park J. Biological effects of blood-brain barrier disruption using a focused ultrasound. Biomed Eng Lett. 2017. doi:10.1007/s13534-017-0025-4.

13. Xu Z, Raghavan M, Hall TL, Chang CW, Mycek M, Fowlkes JB, Cain CA. High speed imaging of bubble clouds generated in pulsed ultrasound cavitational therapy-histotripsy. IEEE. Trans UFFC. 2007;54:2091-101.

14. Park S, Jung U, Lee S, Lee D, Kim C. Contrast-enhanced dual mode imaging: photoacoustic imaging plus more. Biomed Eng Lett. 2017. doi:10.1007/s13534-016-0006-z.

15. Baek H, Pahk KJ, Kim H. A review of low-intensity focused ultrasound for neuromodulation. Biomed Eng Lett. 2017. doi:10. 1007/s13534-016-0007-y. 